\title{
Tratamiento de las contracturas cervicales con colgajo supraclavicular en isla expandido
}

\author{
Treatment of cervical contractures with expanded supraclavicular \\ island flap
}

Carina R. Depasquale ${ }^{1}$, Fabio Coutinho ${ }^{1}$, Gustavo Abrile ${ }^{1}$

\begin{abstract}
RESUMEN
Las secuelas posquemadura en rostro y cuello causan cambios funcionales y aislamiento social. El cirujano plástico reconstructivo utiliza métodos para el tratamiento de estos pacientes, mediante la eliminación de cicatrices y cubriendo el defecto con tejidos adecuados.

El objetivo de la corrección quirúrgica de la contractura de la cicatriz es conseguir la máxima corrección con cambios anatómicos mínimos o nulos, restaurar adecuadamente los tejidos que fueron destruidos en una posición que reduce la contractura lineal limitante.

Diferentes métodos se utilizaron para la reconstrucción de contracturas de cicatrices, con injertos libres de piel, colgajos locales, regionales y colgajos microquirúrgicos, cada uno de los cuales tiene ventajas y desventajas.

El supraclavicular evolucionó hasta el presente, pediculado, en isla, tunelizado y pronto se expandió con éxito, y hoy, un método para la reconstrucción facial completa. Este método puede reducir o eliminar por completo una cicatriz, otorgar color y textura similar que le permite flexibilidad en la zona del cuello que facilita los movimientos tridimensionales.

La técnica consiste en dos etapas como un método que utiliza la prefabricación del colgajo a través de la expansión, y tiene la ventaja sobre otras técnicas que no requiere microcirugía.
\end{abstract}

Palabras claves: contracturas cervicales, colgajo supraclavicular en isla expandido

REVISTA ARGENTINA DE CIRUGÍA PLÁSTICA 2018;24(3):126-137. DOI/10.32825/RACP/201803/0126-0137

\section{INTRODUCCIÓN}

Las contracturas del cuello son debido a quemadura $\mathrm{AB}$ B. En este nivel, la piel es delgada y expuesta a movimientos de flexión. Las secuelas de quemaduras determinan deformidades que van desde una brida mentoesternal a un bloque tumoral cicatriz, lo que limita tanto la región cervical como la cintura escapular. El cirujano plástico reconstructivo utiliza métodos para el tratamiento de estos pacientes, la eliminación de cicatrices y cubriendo el defecto con tejidos adecuados. Hay varios procedimientos reconstructivos utilizados para cubrir el defecto ${ }^{11,28-30}$ y producir resultados satisfactorios funcionales y estéticos como el color, la textura y el espesor de la piel.

Las contracturas que comprometen la cara inferior determinar vectores de tracción, provocando ectropión del párpado, la desviación de la comisura de la boca con el ectropión labio inferior, desviación de las orejas y partes de la nariz, y la dificultad del crecimiento mandibular en niños.

1. Hospital Madariaga. Parque de la Salud. Posadas, Misiones, Rep. Argentina.

$\triangle$ Correspondencia: Dra. Carina R. Depasquale. carinadepasq@hotmail.com

Los autores no declaran conflictos de intereses
El tiempo de aparición de las secuelas es variable, con un promedio de seis meses después de la lesión. Sin embargo, a veces las consecuencias son evidentes a los 3 meses de posiciones forzadas y viciosas. Las secuelas definitivas se manifiestan a los 12 meses cuando la superficie quemada cierra por fibrosis.

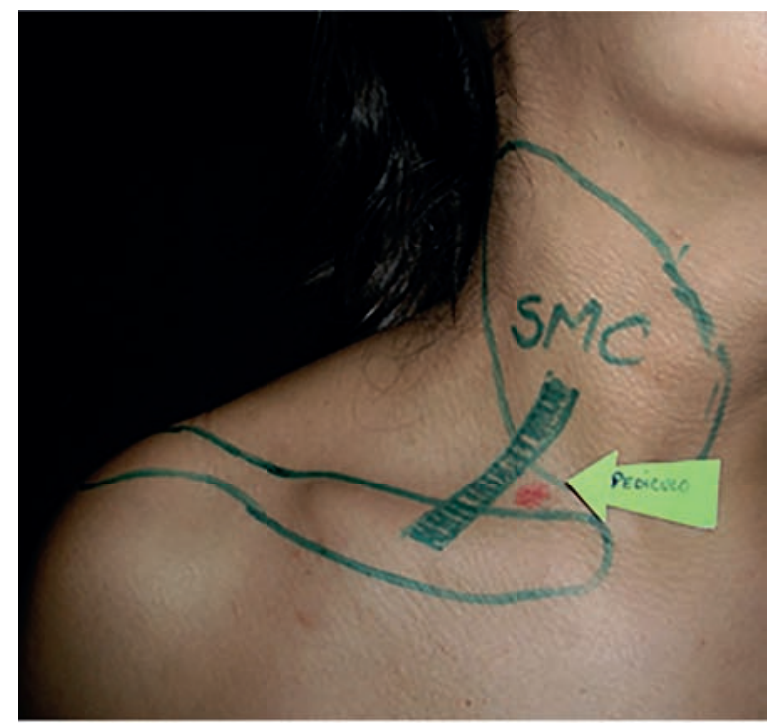

Figura 1. Se observa la localización de la arteria supraclavicular en relación al músculo esternocleidomastoideo, yugular externa y clavícula. 


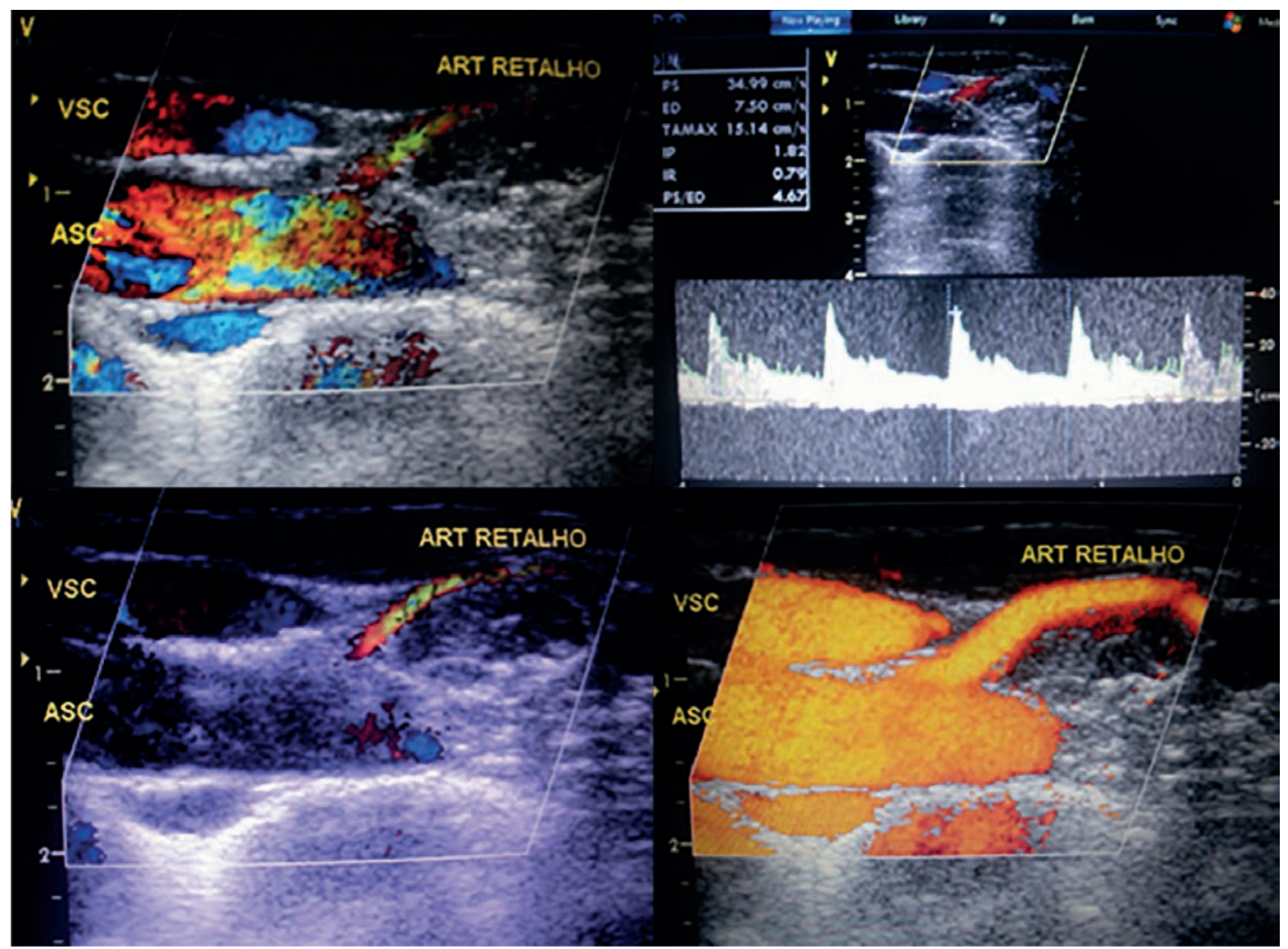

Figura 2.

El principal problema de la contractura es que es inelástica, ubicada en las áreas conjuntas, como el cuello.

El objetivo de la corrección quirúrgica de la contractura de la cicatriz es conseguir la máxima corrección con cambios anatómicos mínimos o nulos, restaurar adecuadamente los tejidos que fueron destruidos en una posición que reduce la contractura lineal limitante.

Diferentes métodos se utilizaron para la reconstrucción de las contracturas cicatrizales, con injertos libres de piel, colgajos locales, regionales y colgajos microquirúrgicos, cada uno de los cuales tiene ventajas y desventajas.

En este trabajo se describe nuestra experiencia en el tratamiento de pacientes con secuela de quemaduras en la cabeza y el cuello mediante el uso del colgajo supraclavicular en isla preexpandida, tunelizada, a través de la implantación de un expansor de tejido en dos tiempos quirúrgicos. Se discuten las ventajas de este método sobre otras técnicas descritas en la literatura.

\section{ANATOMÍA DESCRIPTIVA}

Pallua, en 1999, presentó un trabajo con los estudios anatómicos con disección de cadáveres. En 19 cadáveres, ambas regiones supraclaviculares fueron disecadas 12 a 24 horas después de la muerte. El rango de edad fue de 56 a 84 años, con 12 mujeres y 7 cadáveres masculinos. En todas las muestras, los vasos fueron disecados de triángulo supraclavicular. Los vasos se cortaron en su origen y el diámetro medido. Posteriormente, los angiosomas se identificaron utilizando la inyección de tinta.

\section{Arteria supraclavicular}

En todas las muestras, la arteria subclavia fue expuesta y la arteria cervical transversa disecada. Al 100\% en la arteria supraclavicular nos encontramos con 3 a $4 \mathrm{~cm}$ desde el origen de la arteria cervical transversal. En todos los casos, la arteria se encontró en el triángulo entre límite dorsal del músculo esternocleidomastoideo, la vena yugular externa y la parte medial de la clavícula. El examen reveló que la arteria izquierda iba $3,0 \pm 0,7 \mathrm{~cm}$ por encima de la clavícula a una distancia de $8,2 \pm 1,7 \mathrm{~cm}$ de la articulación esternoclavicular, y alrededor de $2,1 \pm 0,9 \mathrm{~cm}$ dorsal al esternocleidomastoideo. El diámetro medio de la arteria era $0,15 \pm 0,034$ $\mathrm{cm}$ (Figura 1).

\section{Vena supraclavicular}

En todos los especímenes se encontraron dos venas que drenan el colgajo, que no ha sido descrito previamente. En todos los casos, una vena se encontró adyacente a la arteria. Esta vena drena en la vena cervical transversal y tienen un diámetro medio de $0,22 \pm 0,8 \mathrm{~cm}$. La segunda vena es una rama del diámetro de la vena yugular externa de 0,24 $\pm 0,07 \mathrm{~cm}$. En un cadáver, esta segunda vena drenaba en la vena subclavia. 


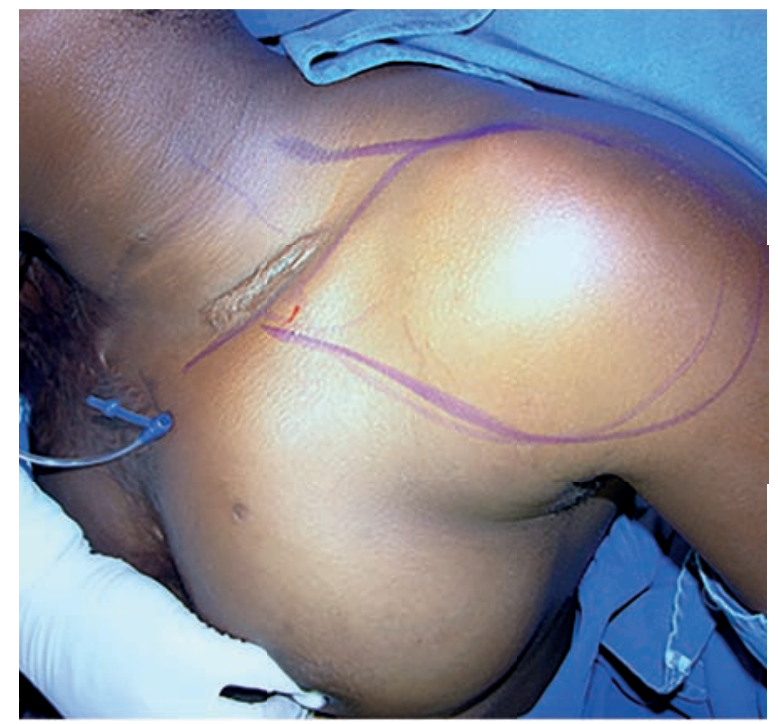

Figura 3. Área de disección del expansor, en plano suprafascial.

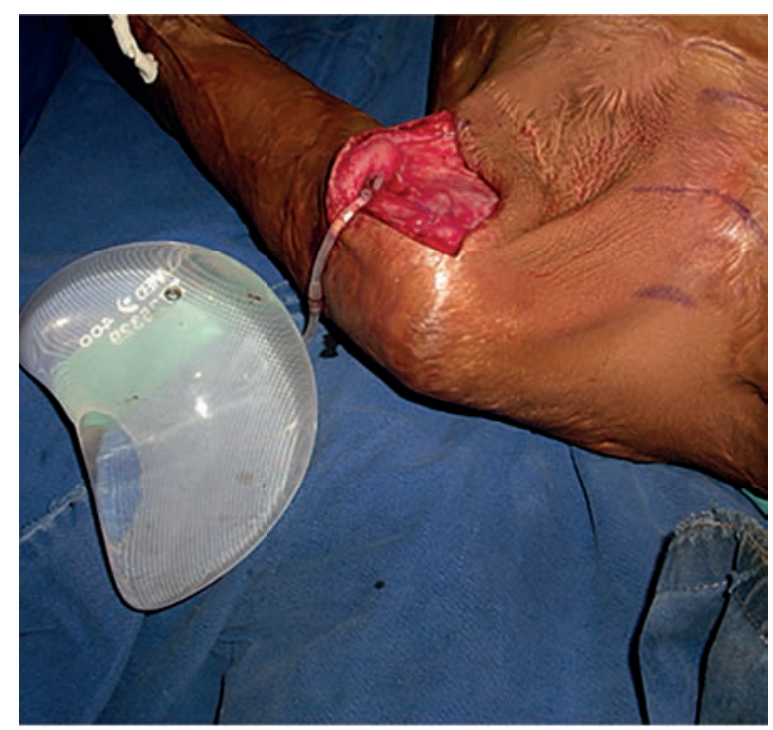

Figura 5. Extracción del expansor y su válvula.

\section{Nervios supraclaviculares}

El nervio supraclavicular está presente principalmente como un grupo de tres a cinco grandes ramas procedentes de la supraclavicular, ganglio del cuello y el hombro. En todas las muestras, se observaron una o dos ramas del plexo cervical a lo largo de las venas en el área del hombro.

\section{Angiosoma de la arteria supraclavicular}

Utilizaron tinta china para mostrar qué área de la piel es abastecida por la arteria supraclavicular. En todos los casos, el territorio vascular se extendía desde la región supraclavicular hasta el hombro. Se puede decir que la parte distal de angiosoma está en la superficie ventral del músculo deltoides. El angiosoma de esta zona oscila entre $10 \mathrm{~cm}$ de ancho y $22 \mathrm{~cm}$ de largo y $16 \times 30 \mathrm{~cm}$. La longitud del angiosoma depende de la altura del cuerpo en una región de 1:6 a 1:8.

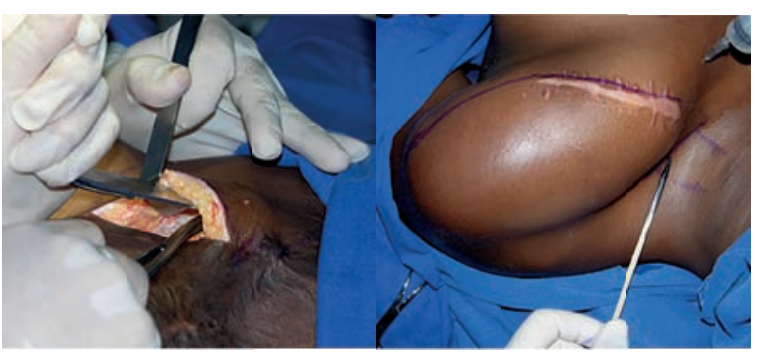

Figura 4. A. Plano suprafascial. B. Marcación del pedículo y expansión perioperatoria.

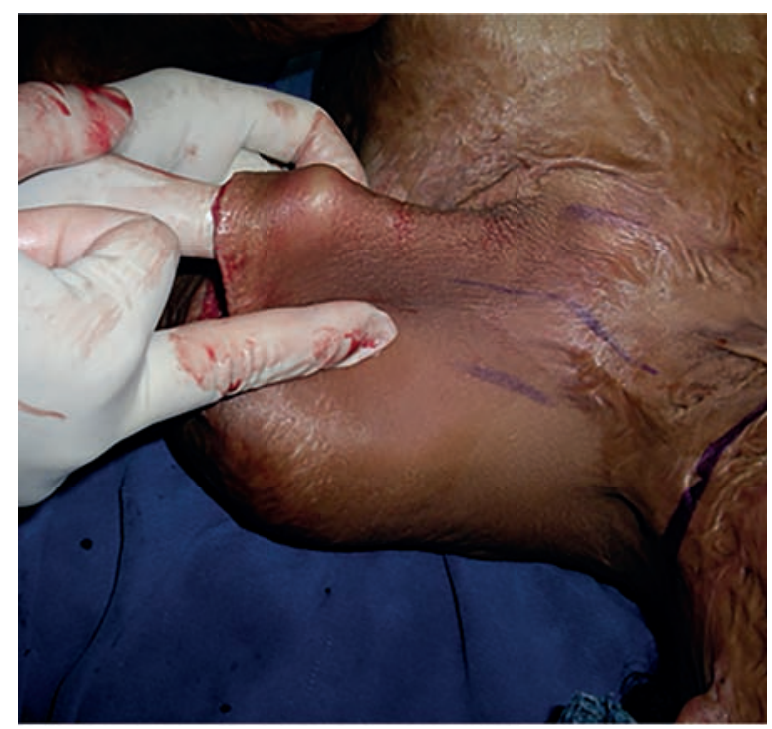

Figura 6. Pinzamiento de la pastilla de piel.

\section{MATERIALES Y MÉTODOS}

Se realizó un trabajo prospectivo de 7 pacientes desde el 2006 al 2014 en el Servicio del Profesor Ivo Pitanguy (3 casos) y el Servicio de Cirugía Plástica del Hospital Madariaga de Posadas (4 casos). Los pacientes presentaban secuelas de quemadura en rostro y cuello y se utilizó colgajo supraclavicular en isla, preexpandido y tunelizado del mismo lado de la secuela y contralateral.

Se realizó eco-Doppler color al colgajo para evaluar la permeabilidad de los vasos del pedículo (Figura 2).

Las cirugías se realizaron en dos tiempos quirúrgicos:

\section{CRITERIOS DE SELECCIÓN:}

Quemaduras presentes en la cabeza y el cuello con diferentes grados de discapacidad funcional por las contracturas de las cicatrices.

Que exista zona dadora para la colocación del expansor (región infraclavicular y el hombro).

PRIMER TIEMPO QUIRÚRGICO

El procedimiento quirúrgico se realiza bajo anestesia general. Todos los pacientes fueron intubados sin broncoscopia, requiriendo la presencia del endoscopista para una posible restricción de acceso debido a la contractura de aire mentoesternal. 


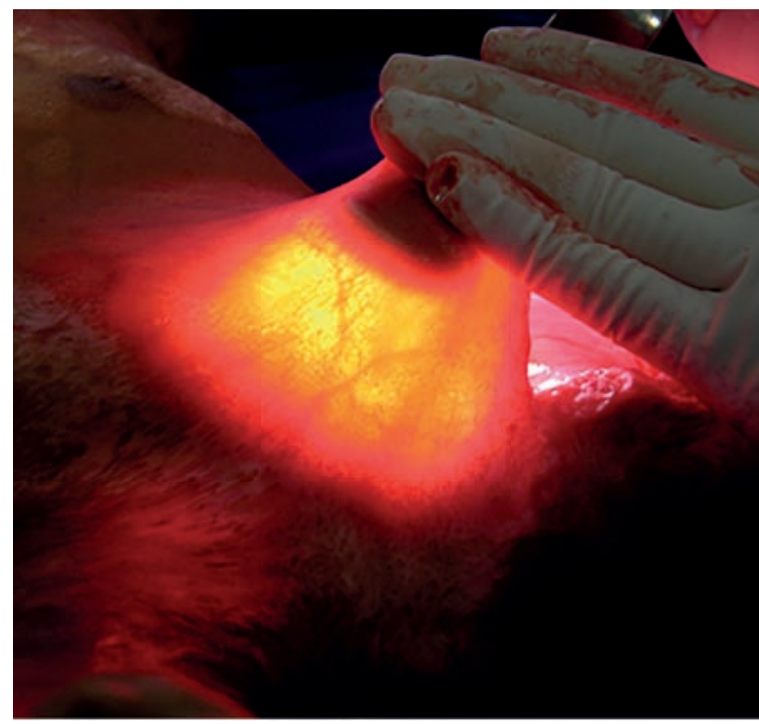

Figura 7. A. Identificación del pedículo por transiluminación.

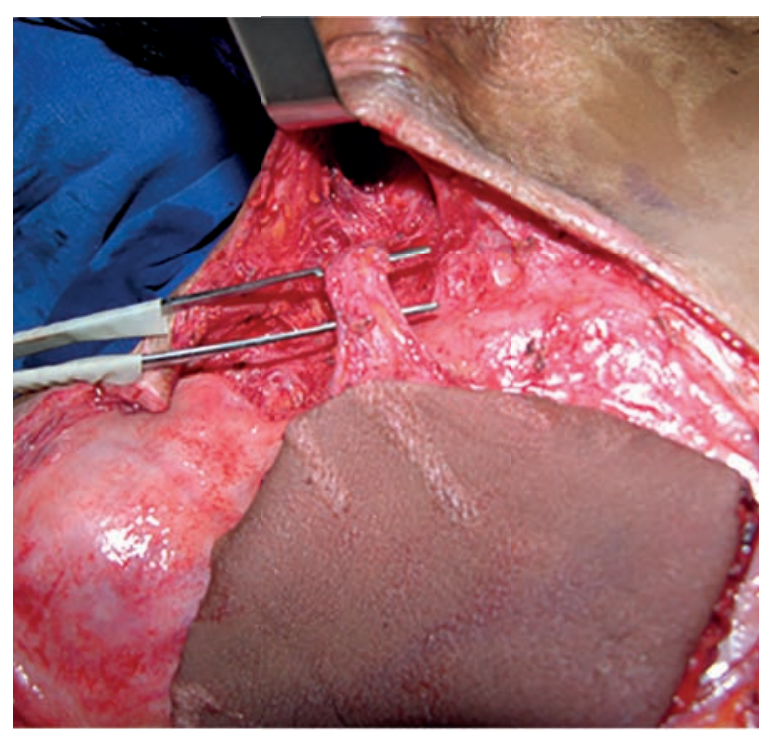

Figura 8. Tunelización del pedículo.

Los pacientes fueron colocados en la mesa de la sala de operaciones en posición supina con la exposición total de la región cefálica a un mejor rendimiento de la técnica quirúrgica. Se lleva a cabo la colocación de la planificación del sitio de la ampliación, situada en la región infraclavicular (incisión, el diseño y el tamaño de la tienda, sitio de colocación de la válvula), dependiendo del tamaño de daño futuro a resecar.

La incisión puede hacerse en las zonas indicadas o no, dependiendo de cada caso particular. Se dibuja con metileno, la incisión principal. El tamaño del expansor define el bolsillo hecho en el plano subcutáneo con tijeras Metzembaum, buscando ir un centímetro más allá del pliegue para un alojamiento mejor del expansor (al expandir se deben evitar pliegues) (Figura 3). Es importante ser cauteloso en el decolamento en la zona cerca del pedículo para evitar posibles daños a la misma. Se realiza la hemostasia exhaustiva. El expansor de tejido se coloca en la región infra-

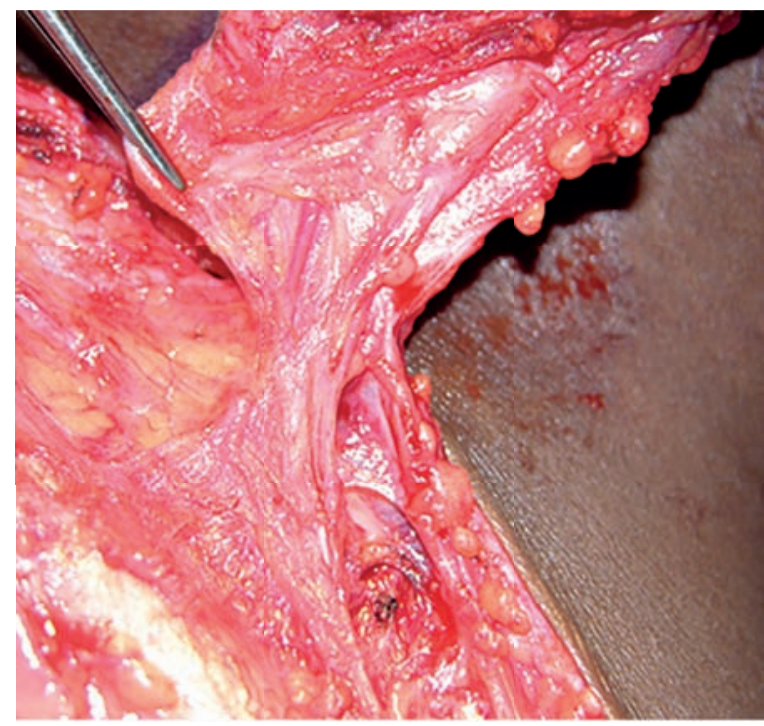

Figura 7. B. Pedículo vascular, arteria y venas.

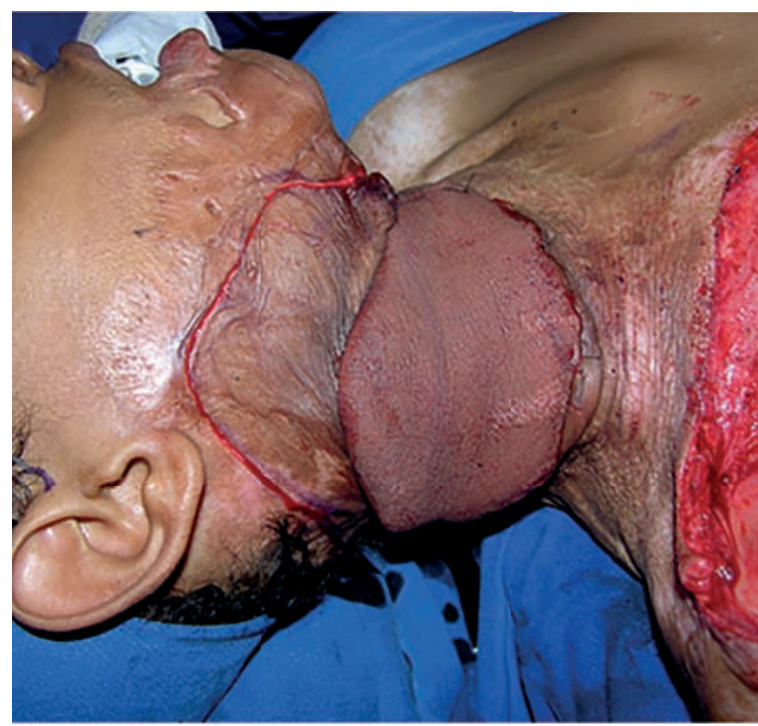

Figura 9. Resección del área quemada y localización del colgajo.

clavicular, el plano suprafascial con la válvula en la región del hombro en la superficie del hueso, lo que facilita la expansión. Como norma de seguridad siempre se hace una prueba en el expansor. En todos los casos se expanden el volumen preoperatorio equivalente a $10 \%$ del expansor.

Utilizamos drenaje de succión, que se retira después de 24 horas. La sutura se hace con mononylon $4.0 \mathrm{El}$ apósito es ligeramente a la compresión.

A los 15 días de la cirugía, las expansiones son semanales, una o dos veces por semana, con aproximadamente el 10\% del volumen total del expansor.

La expansión debe respetar coloración y tensión, así como quejas de dolor. En estos casos, la expansión se disminuye o incluso se desinsufla el expansor (Figura 4).

\section{SEGUNDO TIEMPO QUIRÚRGICO:}

El paciente se somete a anestesia general. Se lleva a cabo la antisepsia y asepsia, la colocación de campos 


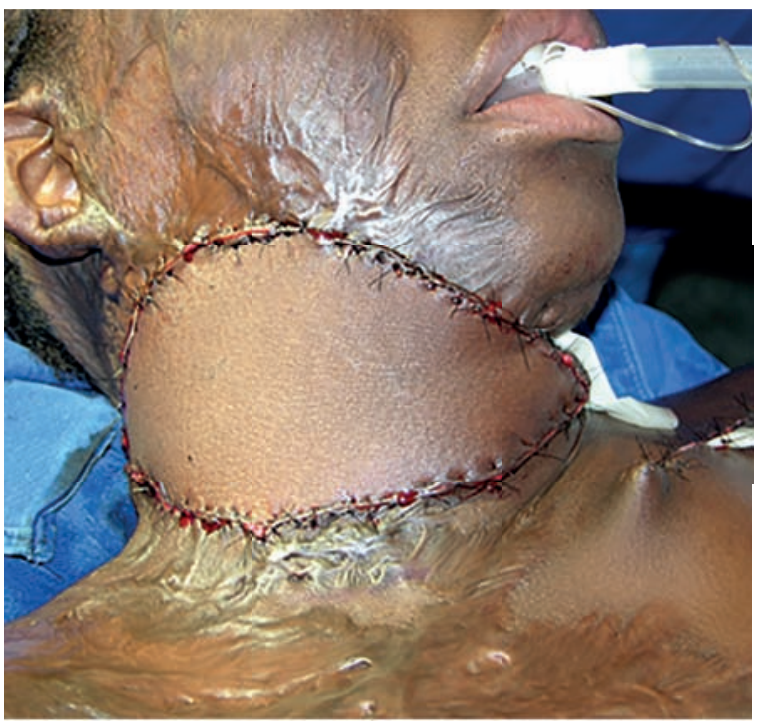

Figura 10. Colocación del colgajo y sutura.

quirúrgicos. El procedimiento es una expansión perioperatoria.

Se dibuja el pedículo teniendo como reparación de un triángulo formado por el límite dorsal de esternocleidomastoideo muscular, la vena yugular externa y el tercio medio de la clavícula. La arteria supraclavicular se eleva a tres centímetros de la arteria cervical transversa tanto a unos ocho centímetros de la articulación esternoclavicular (Figura 4). Se llevó a cabo una cicatriz de la incisión previamente, ya retirado el expansor (Figura 5). Se pinza la isla de piel por medio de una sujeción de la misma con el fin de dejar desollar para su posterior cierre de la zona donante (Figura 6). El enfoque se hace en la dirección proximal a distal para proteger el pedículo. Durante la disección del pedículo es identificado por transiluminación, una vez aislado el pedículo, para diseccionar la tunelización subcutánea (Figuras 7-11). En ese momento la solapa se compone de piel, tejido subcutáneo y el expansor cápsula.

\section{CASOS CLÍNICOS}

\section{CASO 1}

\section{Figuras 12 a 15}

\section{CASO 2}

Figuras 16 a 19

\section{CASO 3}

\section{Figuras 20 a 25}

\section{CASO 4}

Figuras 26 a 31

\section{CASO 5}

Figuras 32 a 34

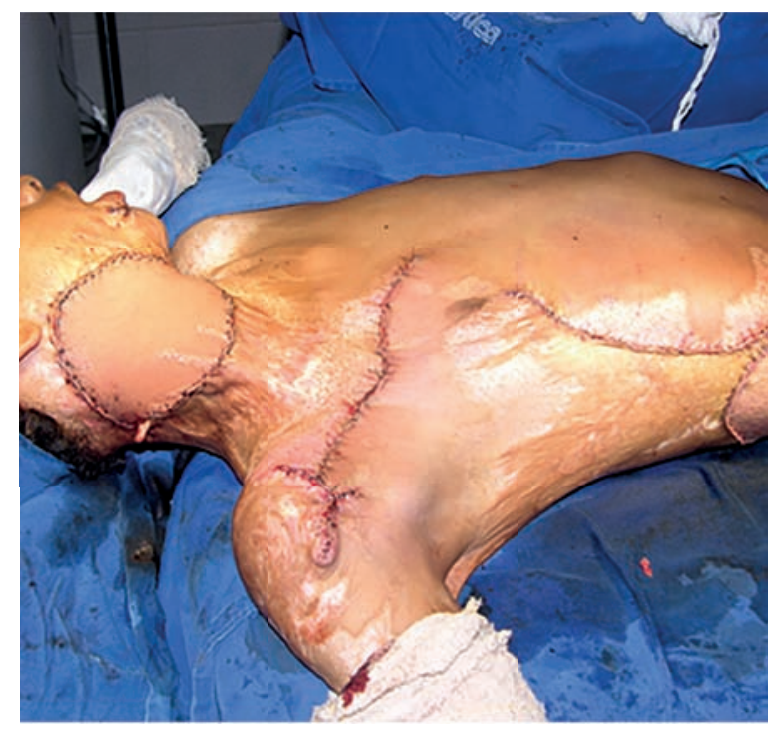

Figura 11. Cierre de zona dadora.

\section{DISCUSIÓN}

Las contracturas por quemaduras en la región cervical anterior tienen particularidades que hacen que sea compleja su corrección y a menudo insuficiente. La restauración de la forma y la función es un gran desafío. La piel del cuello es delgada, y la flexibilidad del cuello predispone a la formación de contracturas, que afectan no solo el movimiento del cuello a la cara inferior y puede, en niños, determinar anormalidades en el crecimiento de la mandíbula.

Actualmente la restauración estética en la cabeza y cuello del paciente quemado todavía representa un reto para la cirugía plástica ${ }^{9-11}$. El método preferido es el que mejor se adapta al caso, en el mejor resultado funcional y estético. Hay diferentes técnicas que utilizan una variedad de colgajos de piel fina para cubrir, con buenos resultados ${ }^{11,28-30}$.

La reconstrucción a inicio de las secuelas de quemadura usaban el autoinjerto como la única alternativa. Actualmente el primer método a ser considerado son los colgajos regionales o locales.

Los principales objetivos para el tratamiento de cicatrices son: eliminar la cicatriz, restauración funcional y evitar la repetición de la cicatriz patológica con una vista estética.

Según la clasificación de las contracturas Achauer mentoesternales, estas son cuatro ${ }^{11,27}$; dependiendo del tamaño de la lesión pueden ser: pequeños defectos cuando se comprometen menos de un tercio de la superficie anterior del cuello; defectos moderados, más de un tercio y menos de dos tercios; defectos graves, compromiso sobre el tercio de la superficie anterior del cuello; y defectos extensos, contractura esternal.

Esta clasificación tiene una importancia didáctica en la planificación de los distintos métodos reconstructivos. El tratamiento recibido por el paciente durante la fase aguda también puede influir en la elección de 


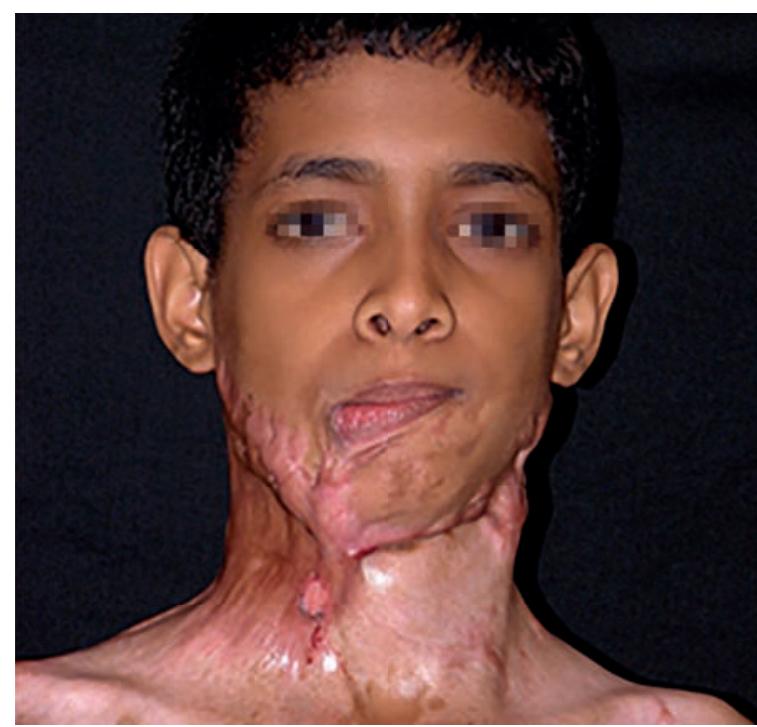

Figura 12.

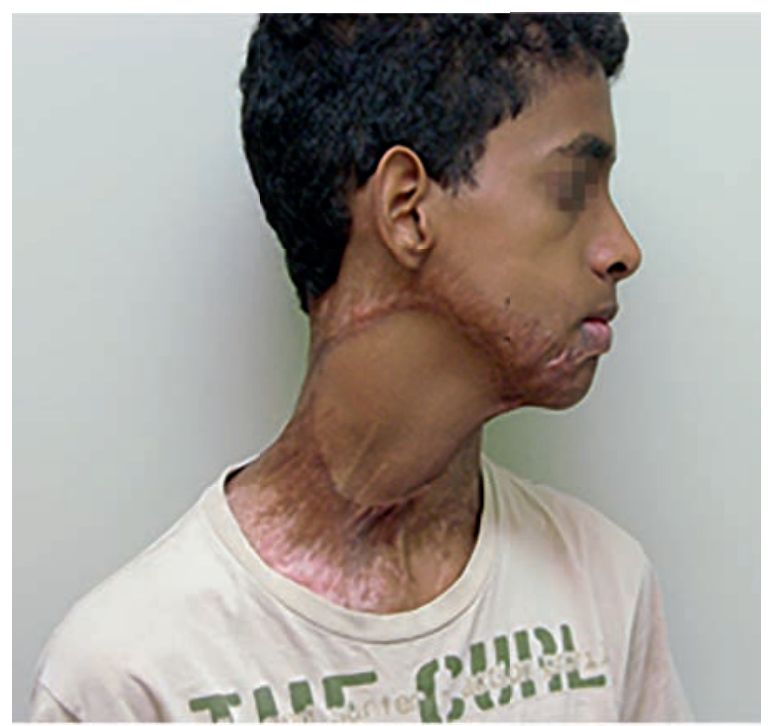

Figura 14.

la técnica de reconstrucción, ya que el sitio del tejido puede haber sido "consumido".

Iwuagwu ${ }^{31}$ (1996) realizó un estudio retrospectivo que compara el uso de injertos parciales y totales de la piel en las contracturas, por quemaduras, en un período de 10 años, donde un total de 126 pacientes tenía 42 las contracturas en la cabeza y el cuello, pensando los mejores injertos totales de piel. La piel parcial mostró una mayor tasa de reoperación (recaí$\mathrm{da}(27,28,32$.

El colgajo local o regional es tomar ventaja tejidos vascularizados y todas las capas a la zona afectada, proporcionando mejores resultados estéticos y funcionales $^{32}$. Entre los colgajos pueden destacarse plástico "Z" y sus variaciones, colgajos prefabricados con la expansión del tejido, colgajos locales y colgajos libres ${ }^{32}$.

Aunque todos estos métodos se pueden utilizar para corregir diferentes tipos de contracturas después de

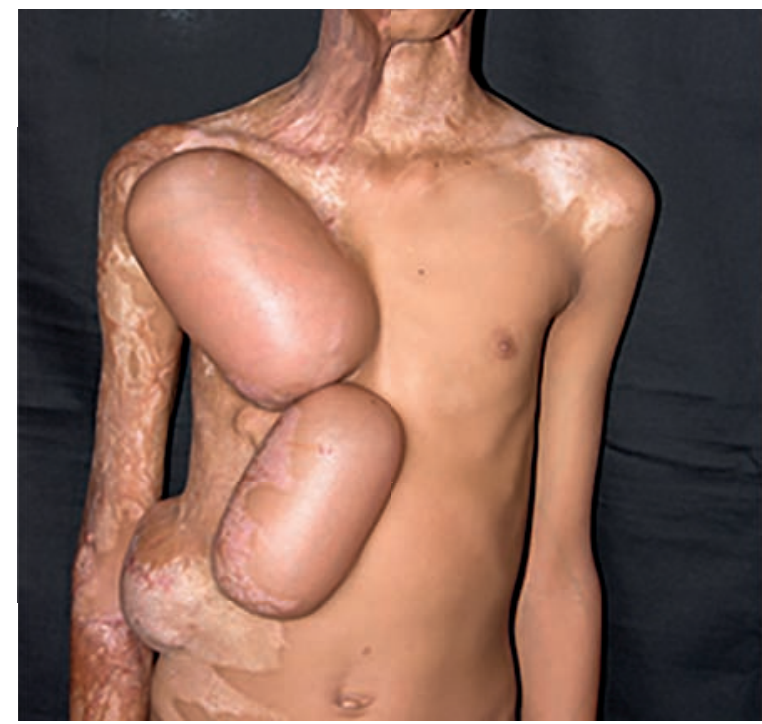

Figura 13.

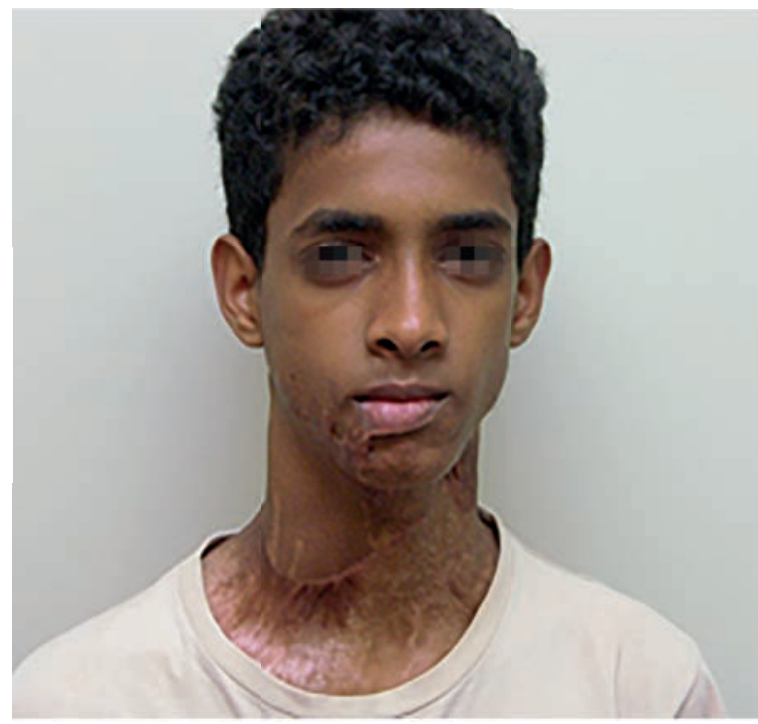

Figura 15.

quemaduras, para el plástico "z" (zetaplastia) es simple, conveniente y eficiente, lo que resulta en un alargamiento máximo de la cicatriz con menos movilidad y superior resolución todavía uno los requisitos que debían cumplirse es tener la piel local suficiente ${ }^{27,32}$ y que la brida no fuera compleja o extensa.

Un colgajo de piel, según Massquelet, puede ser definido de acuerdo con los tres criterios siguientes:

- Anatomía vascular.

- Método de uso.

- Componentes del colgajo. ${ }^{33}$

Vista la vascularización del tejido, se puede distinguir: el colgajo axial estándar, el colgajo con tejido conectivo, el neurocutáneo y el musculocutáneo.

Según el método de uso, se distinguen tres tipos: colgajo libre, colgajo peninsular y colgajo en isla.

Y el criterio final viene dado por los tejidos que lo componen: colgajo fascial, colgajo subcutáneo, colgajos de piel y fasciocutáneos. 


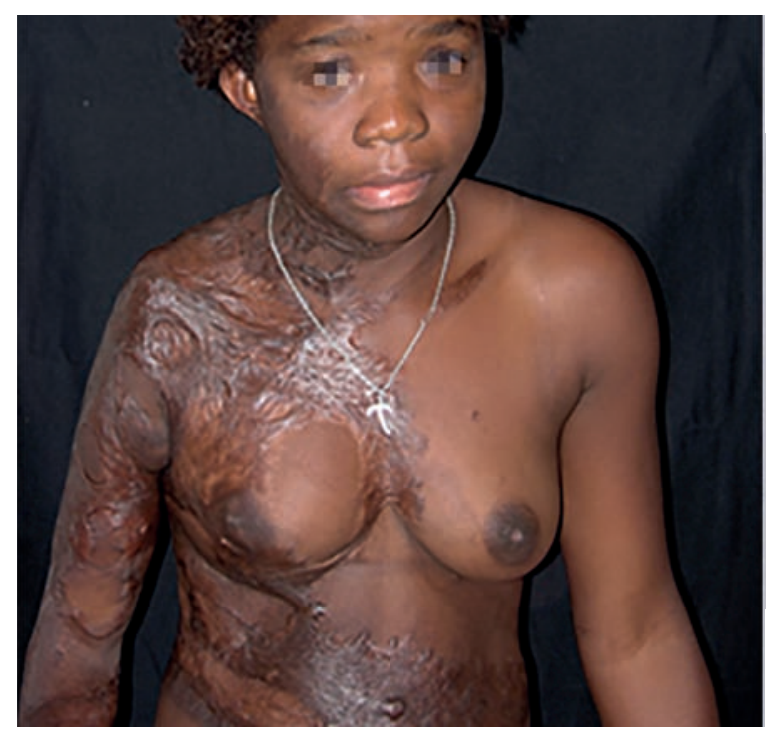

Figura 16.

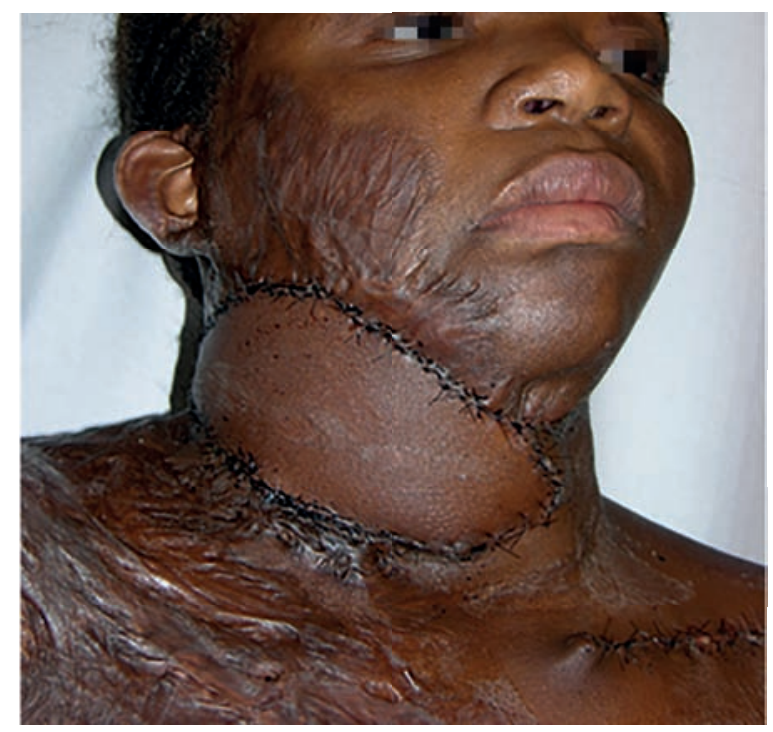

Figura 18.

El pedículo es más fácil, más rápido y más fiable que el colgajo libre. Se requiere, sin embargo, un buen conocimiento de la anatomía vascular.

Los colgajos locales tienen muchas ventajas en el color y la textura, pero a veces los tejidos vecinos están involucrados e impiden su uso.

Los colgajos de piel se remontan a la antigüedad, que se extienden desde la región supraclavicular y el hombro hasta el antebrazo. Fueron descritos por primera vez por Mutter ${ }^{13}$ en 1842 y modificados por Zovickiam ${ }^{14}$ en 1957.

Los primeros estudios anatómicos de estos colgajos fueron hechas por Matheus y Vásconez en $1977^{7}$, que publicó las dimensiones, la vascularización y aplicaciones clínicas patchwork I en la reconstrucción de cabeza y cuello. Los vasos fueron descritos por Matheus y Vásconez: "una rama ascendente de la arteria cervical transversa en la inserción de la clavícula músculo trapecio"; el nombre de la arteria supraclavicular fue dado

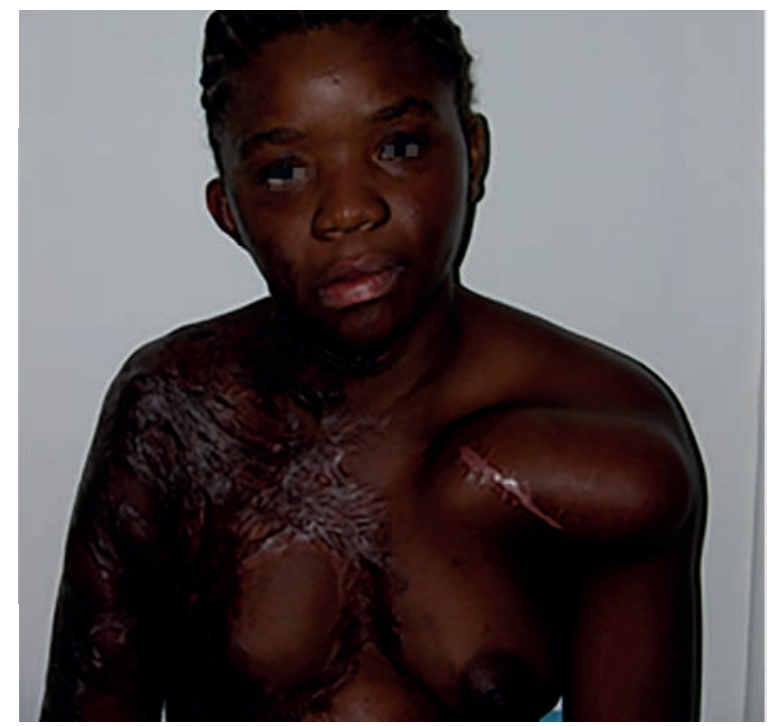

Figura 17.

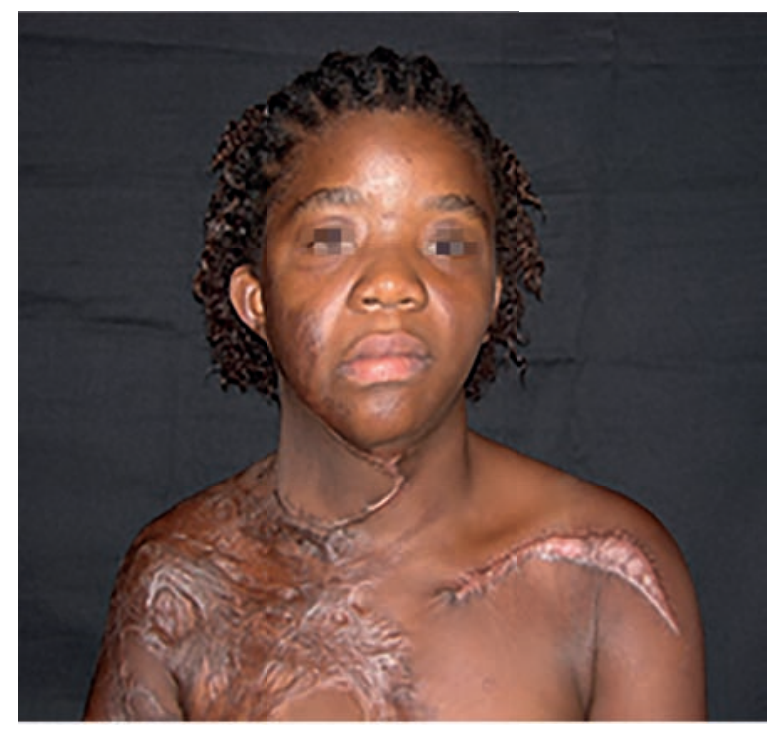

Figura 19.

por Lambert, hubo controversias en el uso de colgajos de piel, ya que su mayor problema es la circulación distal y variables, con alta incidencia de necrosis distal. La aplicación clínica llamada "venta al por menor en charretera" para contracturas mentoesternales fue hecha por Kirschbaum ${ }^{27}$ y Kazanjin y Converse en $1949^{1}$ en el que el mayor inconveniente que presentaba eran: orejas de perro, el aspecto voluptuoso que conduce a la cirugía secundaria al aumento de la morbilidad.

En la región cervical-humeral, los colgajos se pueden dividir en tres categorías de acuerdo con Lambert y Cormack':

1 - Los colgajos de piel (al azar) descritos por Mutter ${ }^{13}$ y luego por Zovickiam ${ }^{14}$; dichos colgajos se utilizaron para contracturas mentoesternales como quemaduras en niños cuando no podían utilizarse colgajos libres.

2- Los tres colgajos musculocutáneos del trapezoide: base superior en la rama de la arteria occipital fue 


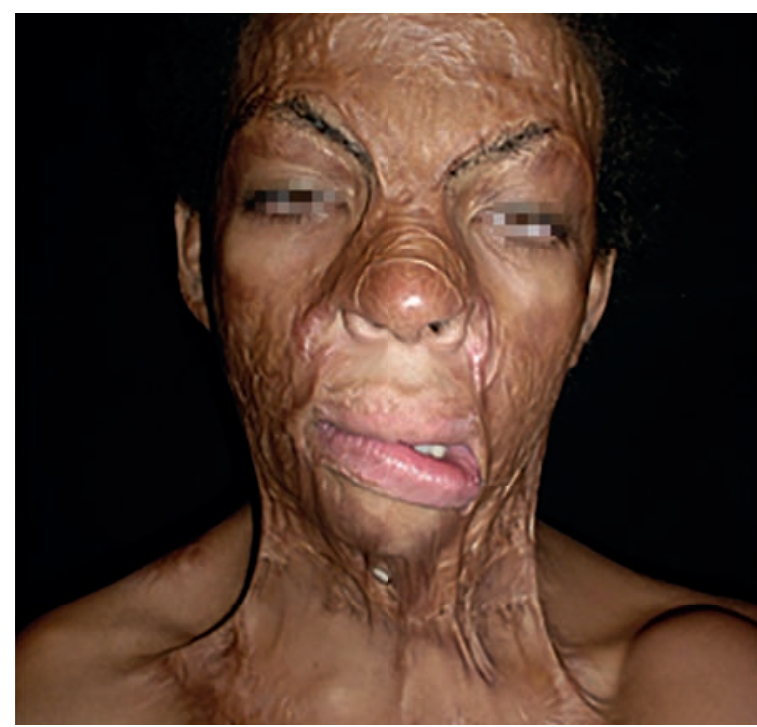

Figura 20.

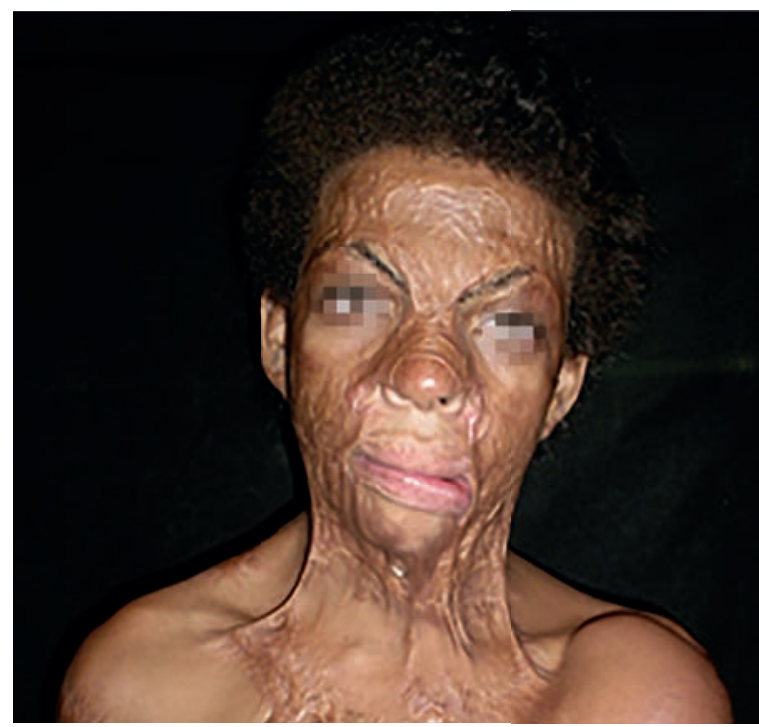

Figura 22.

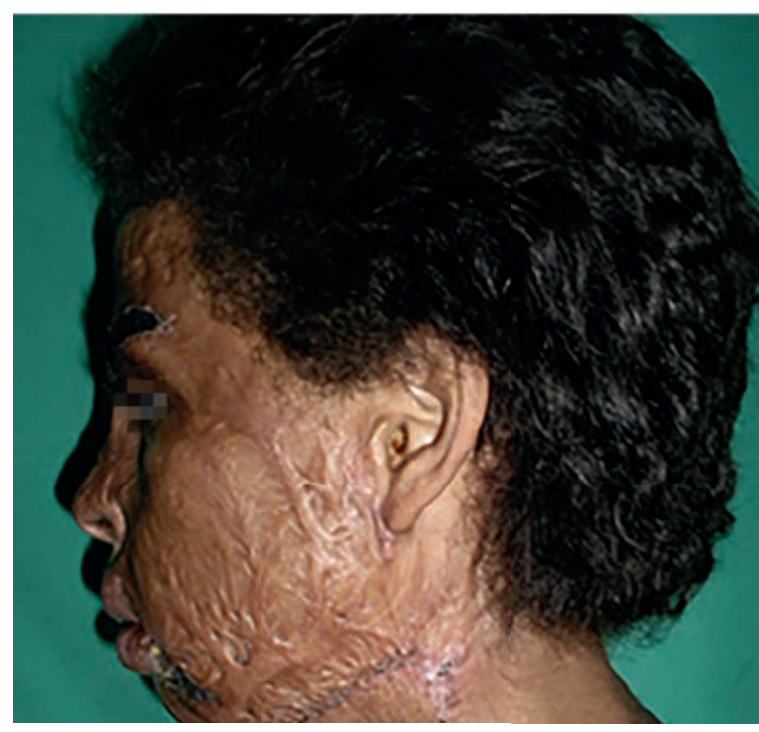

Figura 24.

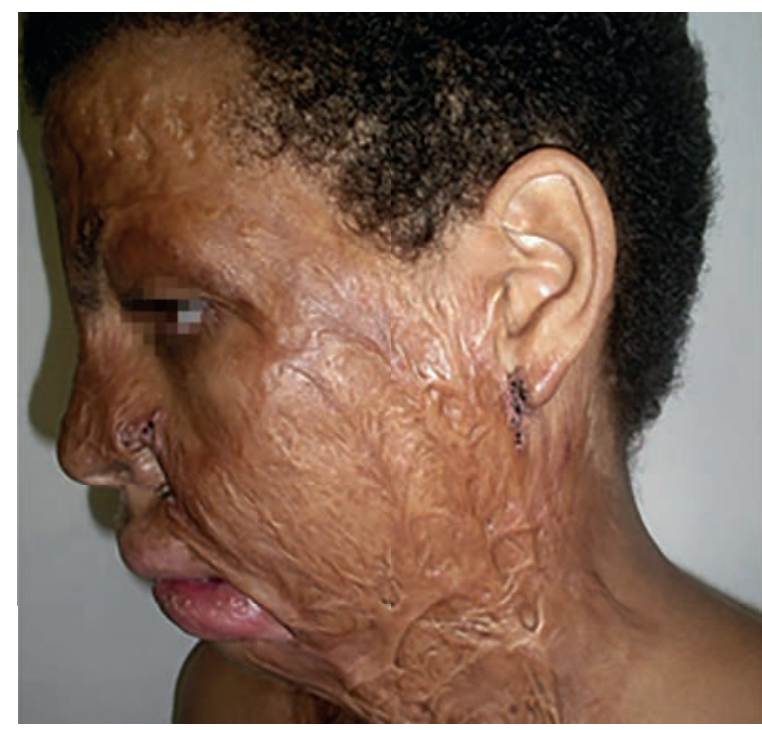

Figura 21.

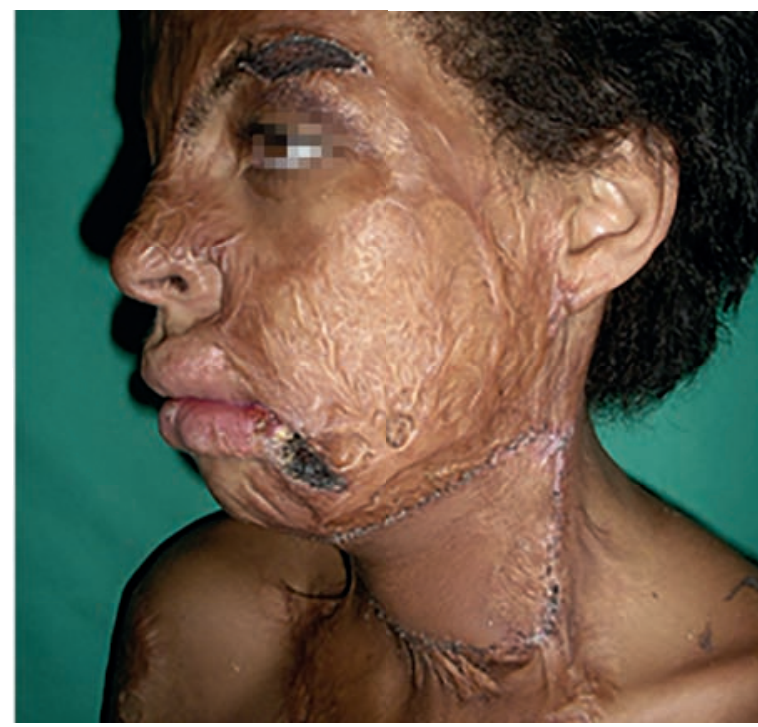

Figura 23

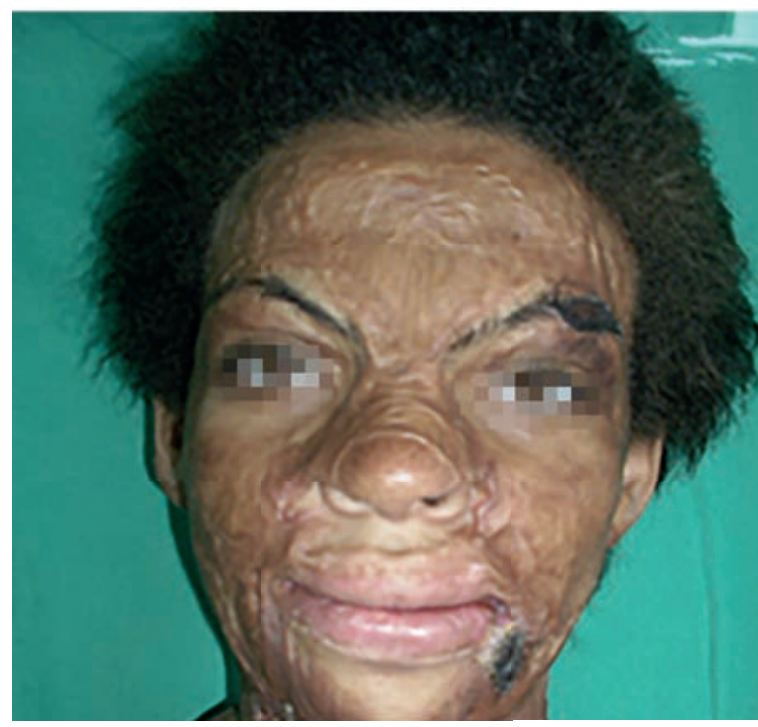

Figura 25. 

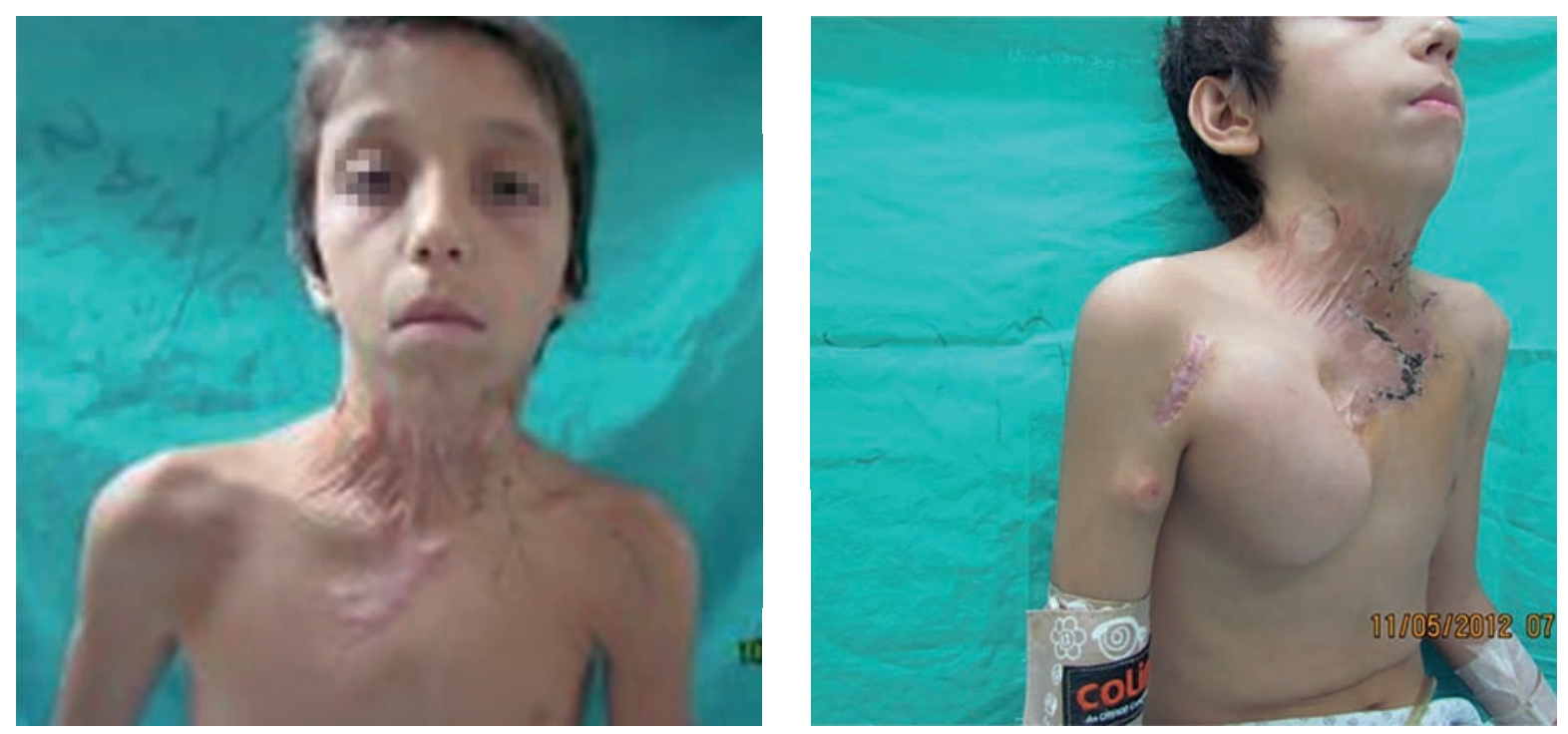

Figura 26.

Figura 27.
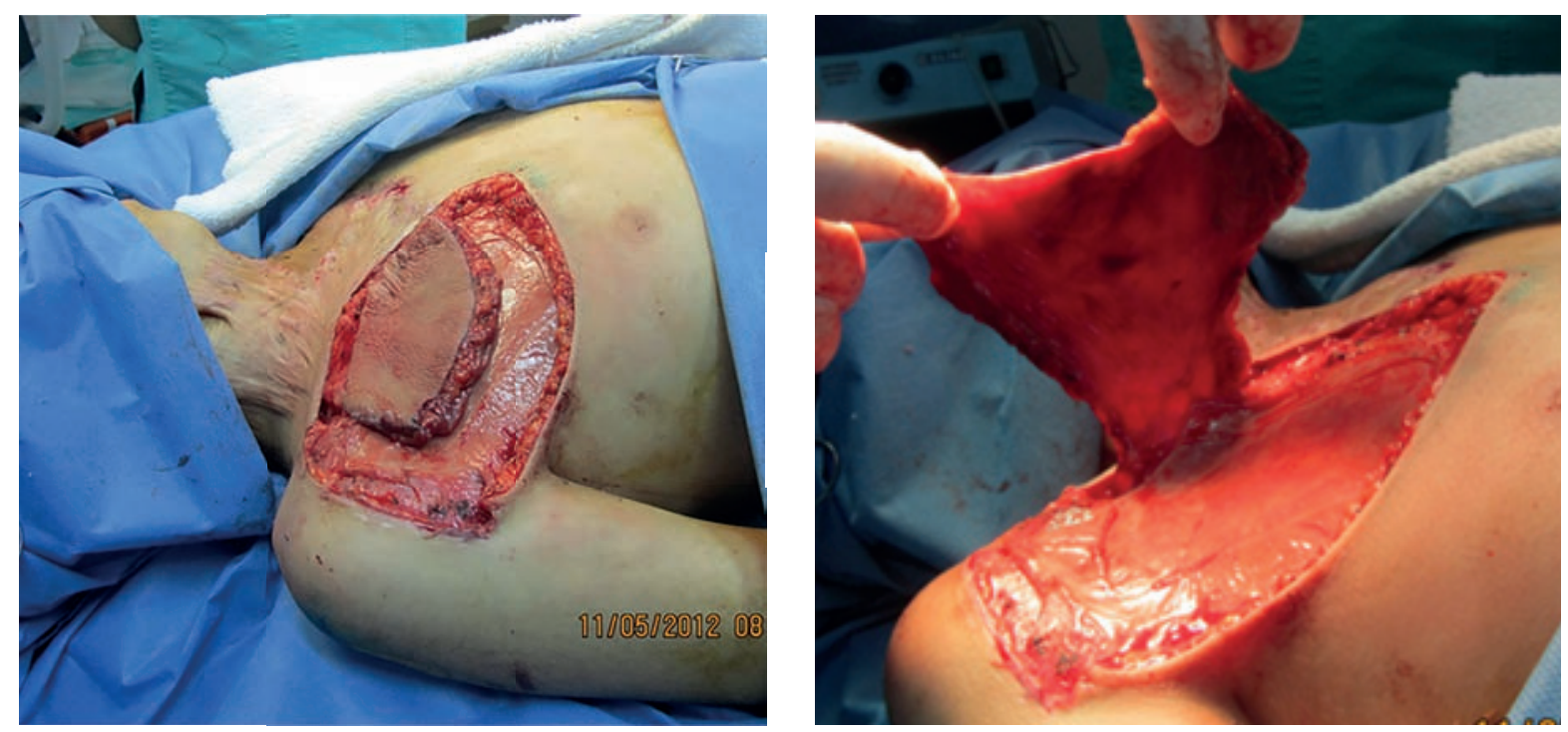

Figura 28.

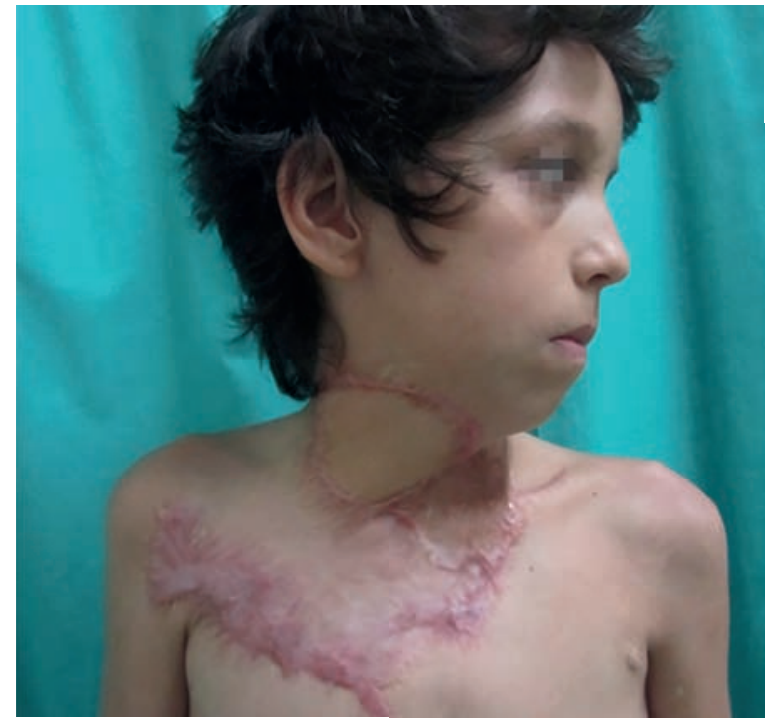

Figura 29.

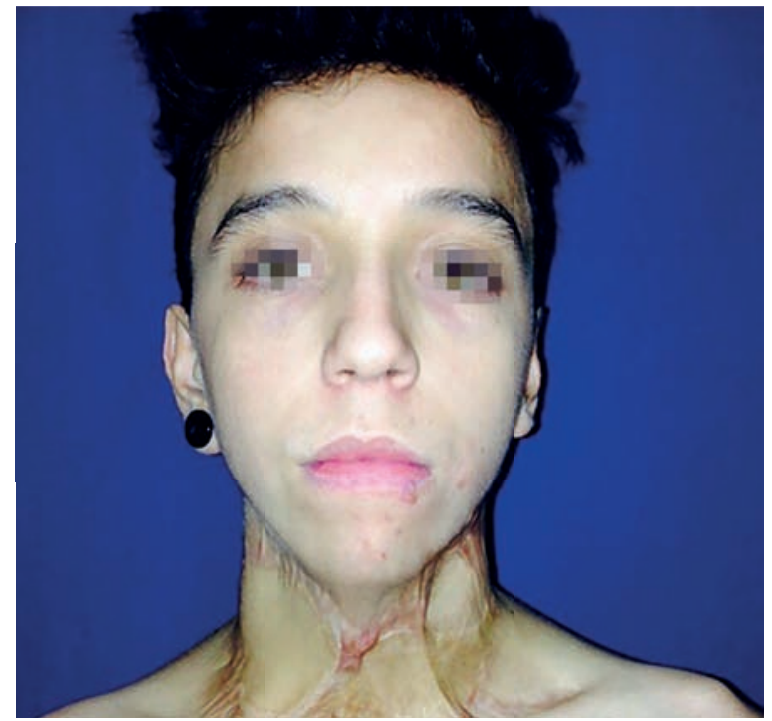

Figura 31. Tres años de posoperatorio 


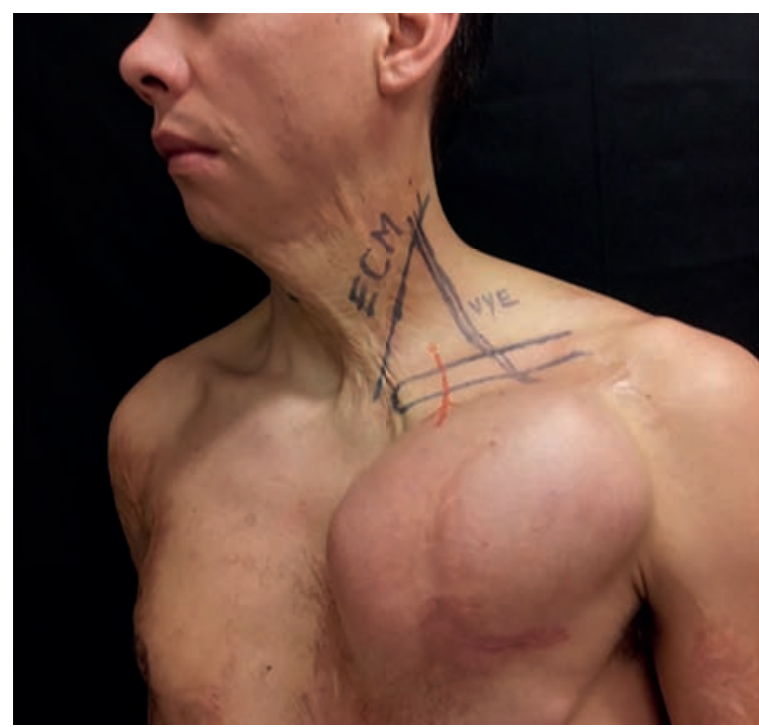

Figura 32

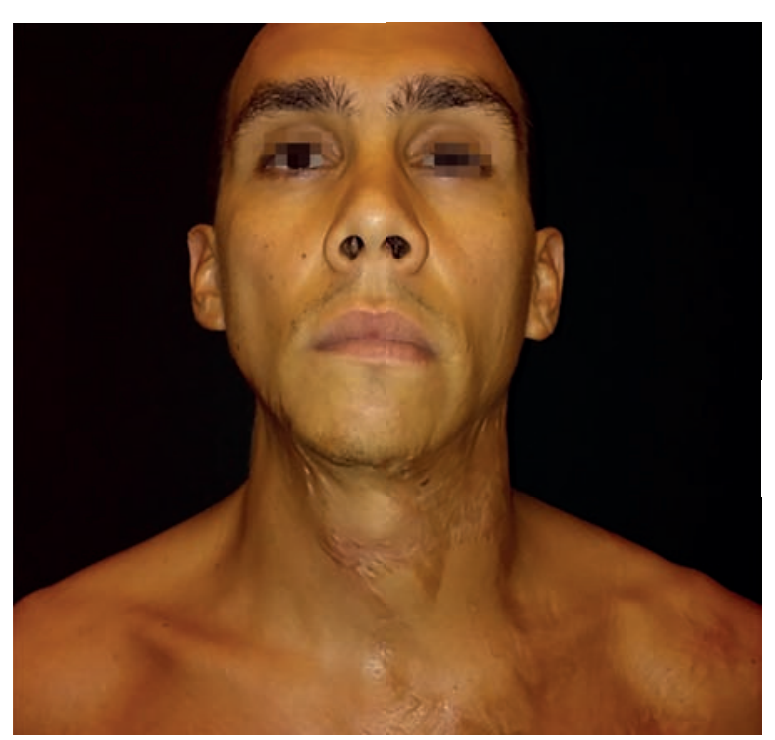

Figura 34.

descrito por McGraw et al., $1977^{15}$, sobre la base de la arteria cervical transversal inferior fue descrito por Nahai 1979 y Mathes ${ }^{16}$ y ramas laterales basado en arteria superficial transversal cervical o arteria cervical superficial descrito por Demergasso $1976^{17}$.

3- El colgajo fasciocutáneo basado en la arteria supraclavicular fue descrito por Lambert ${ }^{16}$ que hizo el estudio anatómico, está otorgando el pedículo axial sobre la base de la arteria supraclavicular. Hay casos con menos frecuencia donde el supraclavicular y la rama de la arteria supraescapular ${ }^{26}$. Una pequeña porción del platisma está incluido en este colgajo.

Un avance importante fue el descubrimiento de la irrigación de la piel de las ramas perforantes musculares, a partir de ahí comenzó a ser utilizado colgajos musculocutáneos para la reconstrucción del cuello, que se utiliza colgajos basados en perforantes del músculo trapecio.

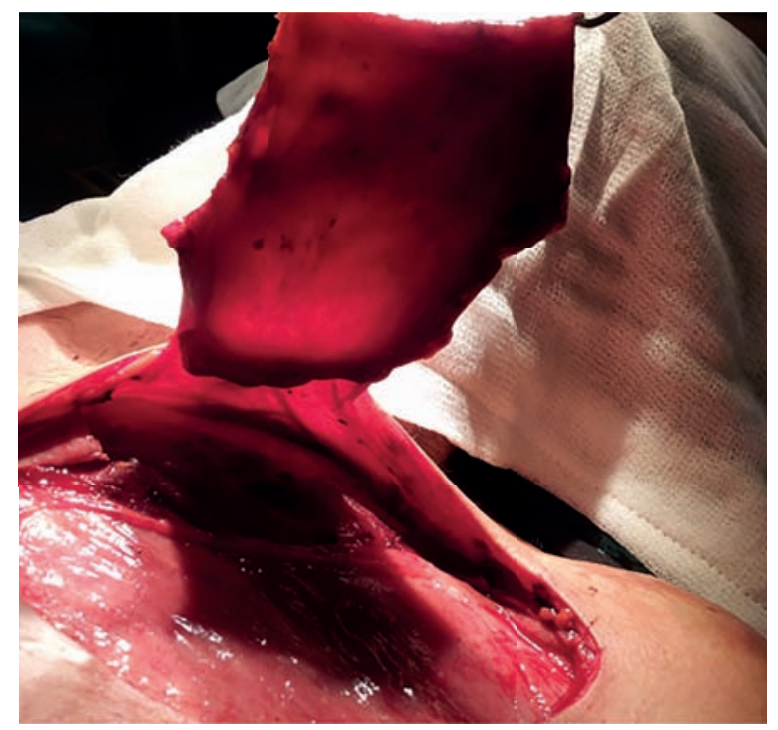

Figura 33.

Lambert ${ }^{4,10}$ describió la circulación fasciocutánea a la piel en la misma ubicación que el Mutter original, pero sobre la base de las ramas de la arteria cervical superficial.

En cuanto a los colgajos libres, Áreas ${ }^{17}$ Americam Society junto de Cirugía Plástica y Recostructive en la Florida, 1970, muestra una reconstrucción de cuello con contractura en una sola operación con colgajo microquirúrgico.

Actualmente los colgajos microquirúrgicos ya no serán la última elección en la reconstrucción, especialmente en áreas como la cabeza y el cuello ${ }^{34}$; se pueden realizar en un solo tiempo quirúrgico, sitios donantes tienen relativamente abundantes y diversas zonas donantes de tejidos con excelente forma, volumen y inervación.

Las desventajas que presenta este tipo de técnica y el alto costo, el equipo quirúrgico capacitado Tsai $\mathrm{Fc}^{35}$ (2003 ( presentó un nuevo método que utiliza preexpansión de colgajos libres cutáneos basados en perforantes, en la que el sitio donante es principalmente cerrado directamente. La mayor ventaja es la prefabricación de grandes colgajos libres para las áreas más grandes de la reconstrucción.

Acta-CHIR Belg ${ }^{36}$ (1998 ( hizo una comparación de tres métodos para la reconstrucción del cuello durante el período de un año, injerto de piel, expansión del injerto, colgajos libre, estudió a 15 pacientes en total cinco con cada método. Juzgado de ser mejor injerto de piel en términos de función, la estética del cuello la movilidad, la elasticidad de la piel, la sensibilidad y la recurrencia de la cicatriz. De acuerdo con las conclusiones de este estudio, para la evaluación de los resultados se hizo en el corto plazo y creemos que con el tiempo estos injertos sufrirán contractura.

Nuestra experiencia con autoinjerto, que todavía se utiliza ampliamente en nuestro servicio, demuestra resultados satisfactorios a corto plazo ponen una tasa de recurrencia alta. Tiene la ventaja de fácil aplicación, bajo costo y facilidad de zona donante la distancia. 
Pallua 1996 utilizado con éxito el colgajo supraclavicular descrito por Lambert. "Ellos aislaron el pedículo " que permite una mayor rotación del colgajo, y se utilizan injerto libre de piel para cubrir el área dadora.

El colgajo supraclavicular isla se ha convertido en un método que se está ampliado para reconstrucción facial completa. Este método puede reducir o eliminar por completo un área quemada mediante la sustitución de un color de la piel similar y textura y permite flexibilidad en la zona del cuello que facilita movimientos tridimensionales.

La técnica consiste en dos etapas como un método que utiliza la prefabricación del colgajo a través de la expansión, y tiene la ventaja sobre otras técnicas que no requiere microcirugía.

El término "prefabricación" se introdujo por $S_{h e n}{ }^{20}$ en 1982. Describió la neovascularización inducida por la introducción de un implante de esta manera para crear nuevo angiosoma al colgajo ${ }^{21-24}$.

La prefabricación requiere múltiples pasos, sin embargo, tiene ventajas porque reduce la morbilidad del sitio dador ${ }^{25}$.

La expansión del tejido se utiliza no solo para mejorar el ancho y el espesor del colgajo , también para aumentar la microvascularización. Como ventaja principal aumenta el tamaño de colgajo entre 14 y $30 \mathrm{~cm}$, y un espesor de 2 a $3 \mathrm{~mm}$.

Actualmente el uso de las expansiones de tejido brinda un resultado estético y funcional más satisfactorio. Utiliza el plano subcutáneo, bajo el platisma en la región supraclavicular o submentón. No hay ninguna descripción de tensión o compresión de la vía aérea.

El paciente y su familia deben ser informados de la deformidad temporal que hace el crecimiento del implante, ya que hay pacientes que rechazan el tratamiento.

Otras complicaciones de la expansión de tejido son las estrías, mal funcionamiento del implante, así como la extrusión, infección, ruptura o perforación, hematoma, seroma, la expansión insuficiente, necrosis de la compresión del tejido.

En nuestra experiencia, hemos observado que la anatomía constante de vasos supraclaviculares hace que esta planificación y la disección sean fáciles, con ventajas como la piel de buena calidad para la reconstrucción de las secuelas de quemaduras en el cuello y la cara sin necesidad de microcirugía. Como desventajas, se citan una cicatriz en el sitio donador de dos tiempos quirúrgicos y el costo de la expansión.

En los últimos años aparecieron en la literatura trabajos sobre el uso de sustitutos dérmicos y epidérmicos. Entre ellos Integra, matriz de regeneración dérmica, desarrollado por Yanas y Burke ${ }^{32}$. Inicialmente se utilizó en la cara quemadura aguda y ahora también en la fase tardía. Tiene dos capas, el exterior y el interior de silicona formada de colágeno bovino. Su principal ventaja es que proporciona una lista para recibir neoderme un autoinjerto muy delgada $(0,04-0,06 \mathrm{~cm}$ de espesor) sin la contracción indeseable de la misma, con un resultado positivo del punto de vista práctico y estético. Como desventaja del procedimiento, se lleva a cabo en dos etapas, es necesario autoinjerto, y el alto costo.

\section{CONCLUSIÓN}

El colgajo supraclavicular en isla expandido demuestra según la literatura y nuestra experiencia ser versátil y fácil de realizar. Se puede utilizar en la reconstrucción de cabeza y cuello con excelentes resultados estéticos y funcionales. La técnica quirúrgica es sencilla, dada la anatomía constante de los vasos implicados. Se compone de dos etapas, usando el método como la prefabricación del colgajo a través de la expansión, y tiene la ventaja sobre otras técnicas que se puede hacer en los centros de baja complejidad a bajo costo.

\section{BIBLIOGRAFÍA}

1. Kazanjian VH, Converse JM. The surgical treatment of facial injuries. Baltimore: Williams \& Wilkins, 1949.

2. Demergasso F, Piazza MV. Trapezium miocutaneus flap in reconstructive surgery for head and neck cancer: An original technique. Am. J. Surg. 138: 533, 1979.

3. Mathes SJ, Nahai F. Clinical Atlas of muscle and musculocutáneus flaps St. Louis: Mosby 1979.

4. Mathes DJ,Nahai F. Clinical applications for muscles, anatomy \& Technique. New York: Churchill Livinstone, 1997. Pp. 10-12.

5. Conmark GC, LambertyBGH. The anatomical vascular basis of the auxiliary fasciocutaneus pedicle flap. Br. J. Plast. Surg. 36: 125, 1983.

6. Matheus SJ, Vasconez LO. The cervico-umeral flap. Plast. Reconstr. Surg. 61:7, 1978.

7. Blevins PJ, Luce EA. Limitations of the cervico-umeral flap en head and neck reconstructions. Plast. Reconstruct. Surg. 66: 220, 1980.

8. Lamberty BGH, Conmark GC. Misconceptions regarding the cervico-humeral flap. Br. J. Plast. Surg. 36: 60, 1983.
9. Pallua N, Machens HG, Remekampff O, Becker M, Berger A. The fasciocutaneous supraclavicular artery island flaps for realizing post-burn mentoesternal contractures. Plast. Reconstruct Surg. 99: 1879. 1997.

10. Pallua N, Noah EM. The tunneled supraclavicular island flap: an optimized technique for head and neck reconstruction. Plast. Reconstruct Surg. 105: 842, 2000.

11. Pallua N, Von Heimberg D. Pre- expanded ultra-thin supraclavicular flaps for (full) face reconstruction with reduced donor-site morbility and without the need for microsurgery. Plast. Reconstr. Surg. 1877. 2004.

12. Mutter TD. Case of deformity from burns relieved by operation. Am. J. Med. Sc. 4: 66, 1842.

13. Zovickian A. Pharyngeal fistulas: repair and prevention using mastoid - occipit based shoulder flaps. Plast. Reconstr. Surg. 19: 355, 1957.

14. Mc Crow JB, Dibell DG, Carraway JH. Clinical definition of independent miocutaneous vascular territories. Plast. Reconstr. Surg. 60: $341,1977$. 
15. Mathes C, Nahai F. Clinical Atlas of Muscle and fasciocutaneous Flaps. St. Louis: Mosby, 1979

16. Demurgasso F, Piazza M. Trapezius myocutaneous flaps in reconstructive surgery for head and neck cáncer: an original technique. Am. J. Surg. 138: 533, 1979.

17. Harii K. Microvascular transfer of the groin flaps to the neck. Presented at the annual meeting of the American Society for Plastic and Reconstructive surgery. Hollywood. 1973.

18. Lamberty BGH, Cormack GC. Misconceptions reagarding the cervico-humeral flap. Br. J. plast. Surg. 36: 60, 1983.

19. Lamberty BBH. The supraclavicular axial - patterned flaps.. Br. J. Plast. Surg. 32: 207, 1979.

20. Shen TY. Microvascular transplantation of prefabricated free thig flap (letter) Plast. Rector. Surg. 69: 568, 1982.

21. Khouri RK. Facial reconstruction with pre-fabricated induced expandid (PIE) supraclavicular skinflaps. Plast. Recostr. Surg. 95: 1007. 1995.

22. Teot L. Cherenfant E. Prefabricated vascularised supracalvicular flap for face resurfacing after post burns scarring. Lancet 355: 1695. 2000

23. Barthelemy I, Martin I, Pre-fabricated superficial temporal fascia flap combined with submental flap en noma surgery. Plastic. Reconstr. Surg. 109: 936. 2002.

24. Pribaz JJ, Fine NA. Pre-fabricated and pre-laminated flaps for head and neck reconstruction. Plast Surg. 28: 261, 2001.
25. Heinz TR, Cowper PA, Levin LS. Microsurgery costs and Outcome Plast. Recostr. Surg. 104: 889, 1999.

26. Di Benedetto G. From the "charretera" to the supraclavicular fascial island flap: revisitation and further evolution of a controversial flap. Plast. Recostruc. Surgery. Janeiro-70-76. 2005.

27. Achauer BM. Burn reconstruction. Plastic Surgery. Cap 29: 425-446. ed. 2000.

28. Angrigiani C. Total face reconstruction whit one free flap. Plast. Reconstruc. Surg. 99: 1566, 1997.

29. Angrigiani C. Aesthetic microsurgical reconstruction of anteriorneck burn deformities. Plast. Reconstruc. Surg. 93: 507, 1994.

30. Zimman OA. Reconstruction of the neck whit two rotation-advancement platysma myocutaneous flaps. Plast. Reconstr. Surg. 103: 1712,1999

31. Iwuagwu FC. The use of skin grafts in postburn contracture release: 10-year review. Past. Rector. Surg. 103: 1198-1204, 1999.

32. Leonardi DF, Tostes F. Piccolo N. Seqüelas de quemaduras. Cirurgia Plástica. Cap 84.: 869-882. Ed 2005.

33. Masquelet AC. Atlas colorido de retalhos na reconstrução de membros. Cap 2: 14-17. Ed 1997.

34. Nassif T. Microcirurgia, princípios gerais. Cirurgia Plástica. Cap 22: 185-191. ed. 2005

35. Tsai FC. Burns. Pubmed. 14636763. 29 (8): 845-848. 2003.

36. Acta-Chir-Belg. ISSN. 0001-5458. Janeiro, fevreiro. 98(1): 5-9.1998. 